\title{
Lipid Replacement Therapy Drink Containing a Glycophospholipid Formulation Rapidly and Significantly Reduces Fatigue While Improving Energy and Mental Clarity
}

\section{Rita R. Ellithorpe, ${ }^{1}$ Robert Settineri, ${ }^{2}$ Cyndee A. Mitchell, ${ }^{1}$ Brett Jacques, ${ }^{1}$ Erin Ellithorpe, $\mathrm{BA}^{1}$ and Garth L. Nicolson, ${ }^{3}$ *}

${ }^{1}$ Tustin Longevity Center, Tustin, California 92780, USA

${ }^{2}$ Sierra Research, Irvine, California 92606, USA

${ }^{3}$ Department of Molecular Pathology, The Institute for Molecular Medicine, Huntington Beach, California 92647, USA

Corresponding author: Prof. Garth Nicolson, Office of the President, The Institute for Molecular Medicine, P.O. Box 9355, S. Laguna Beach, CA 92652

Submission date: July 26, 2011; Acceptance date: August 26, 2011; Publication date: August 29, 2011

\begin{abstract}
:
Background: Fatigue is the most common complaint of patients seeking general medical care and is often treated with stimulants. It is also important in various physical activities of relatively healthy men and women, such as sports performance. Recent clinical trials using patients with chronic fatigue have shown the benefit of Lipid Replacement Therapy in restoring mitochondrial electron transport function and reducing moderate to severe chronic fatigue.
\end{abstract}

Methods: Lipid Replacement Therapy was administered for the first time as an all-natural functional food drink $(60 \mathrm{ml})$ containing polyunsaturated glycophospholipids but devoid of stimulants or herbs to reduce fatigue. This preliminary study used the Piper Fatigue Survey instrument as well as a supplemental questionnaire to assess the effects of the glycophospholipid drink on fatigue and the acceptability of the test drink in adult men and women. A volunteer group of 29 subjects of mean age $56.2 \pm 4.5$ years with various fatigue levels were randomly recruited in a clinical health fair setting to participate in an afternoon open label trial on the effects of the test drink.

Results: Using the Piper Fatigue instrument overall fatigue among participants was reduced within the 3 -hour seminar by a mean of $39.6 \%(p<0.0001)$. All of the subcategories of fatigue showed significant reductions. Some subjects responded within 15 minutes, and the majority responded within one hour with increased energy and activity and perceived improvements in cognitive function, mental clarity and focus. The test drink was determined to be quite acceptable in terms of taste and appearance. There were no adverse events from the energy drink during the study. 
Functional Foods in Health and Disease 2011, 1(8):245-254

Page 246 of 254

Conclusions: The Lipid Replacement Therapy functional food drink appeared to be a safe, acceptable and potentially useful new method to reduce fatigue, sustain energy and improve perceptions of mental function.

Keywords: functional food drink, NT Factor®, NT Factor Lipids®, fatigue, mitochondrial function, mental function

\section{Background:}

Chronic or intractable fatigue that is not reversed by sleep occurs naturally during aging and in many degenerative diseases $[1,2]$. It is the most common complaint of patients seeking general medical care in North America [1, 3].

Fatigue or loss of energy is quite a common event in most adults. Fatigue is associated with many normal activities, such as sports and physical training as well as certain occupations [4-6]. To combat fatigue various strategies have been employed, including nutritional supplements and "energy" drinks that usually contain high concentrations of sugars and stimulants, such as caffeine (1,3,7-trimethylxanthine) and herbal extracts $[7,8]$.

\section{Introduction:}

Fatigue is usually understood as a loss of overall energy and inability to perform tasks without exertion. At the cellular level fatigue is related to cellular energy systems found primarily in the cellular mitochondria $[2,9,10]$. Damage to mitochondrial components, especially mitochondrial membranes, occurs mainly by oxidation, and this can result in increased ion leakage across mitochondrial membranes and impairment in the ability of mitochondria to produce high-energy molecules needed for daily activities [2, 9-11].

During normal physical activity and in chronic diseases Reactive Oxygen and Nitrogen species (ROS/RNS) are produced that can cause oxidative stress and cellular damage, resulting in oxidation of lipids, proteins and DNA [11-13]. Important targets of ROS/RNS damage are mitochondria and cell membranes, mainly their phospholipids components [10, 11, 12]. Similar damage occurs in fatiguing illnesses, such as chronic fatigue syndrome, cancer and other diseases [14,15]. In addition, mitochondrial function is known to decrease during aging, and its decline has been implicated in sarcopenia [16].

Recent clinical trials have shown the effectiveness of Lipid Replacement Therapy (LRT) plus antioxidants in the treatment of certain clinical disorders and conditions, such as chronic fatigue and chronic fatigue syndrome $[2,9,10]$. LRT is a dietary approach to replace damaged cellular lipids with undamaged (unoxidized) lipids to ensure proper function of cellular structures, such as cellular and organelle membranes [9, 10]. LRT can result in the cellular delivery of unoxidized, undamaged membrane glycophospholipids in order to replace damaged lipids and restore function to oxidized, damaged cellular membranes. LTR has proven to be an effective method to prevent ROS/RNS-associated changes in function and for use in the treatment of various clinical conditions [2, 9, 10, 17].

"Energy" drinks have been proposed as a way to quickly combat fatigue and provide the perception of energy, but most contain sugar and stimulants like caffeine or herbs [7,8]. Here we report on an "energy" drink containing membrane phospholipids and glycophospholipids 
without added sugar, caffeine or herbal stimulants to reduce fatigue and improve perceptions of mental function.

\section{Subjects and Methods:}

Subjects. Participants were adult male $(n=8)$ and female $(n=21)$ volunteers who attended an afternoon health seminar at a medical clinic. Subjects were asked if they used any prescription medications to see if this might exclude them from the study, as determined previously [17]. The mean age of the participants was $56.2 \pm 4.5$ years (male, $54.6 \pm 4.5$ years; female, $56.95 \pm 3.1$ years).

Study Design. Subjects signed an informed consent document and were admitted into an open label study with measurable fatigue levels (2-6) using the Piper Fatigue Scale (PFS). This is a validated instrument for measuring fatigue using a survey questionnaire $[18,19]$. In addition, a supplemental survey form was used to determine participant acceptance of the product being tested and other parameters. After filling out the PFS form, participants were given instructions to take one two-ounce $(60 \mathrm{ml})$ liquid drink of the membrane glycophospholipid test supplement (Table 1). All subjects repeated the PFS assessment at the end of the three-hour seminar without access to their previous scores, and they also filed out the Supplemental Survey Form (Table 2). All of the forms were checked for verification, completion and scoring accuracy [20]. There were no ethical questions raised on the design or execution of the study.

Table 1. Test Supplement (NT Factor Lipids®)

\begin{tabular}{ccc}
\hline & Amount & $\%$ \\
Component & Per & Daily \\
& Serving & Value* $^{*}$ \\
\hline NT Factor Lipids ${ }^{\#}$ & & $* *$ \\
Total Fat & $600 \mathrm{mg}$ & 0.40 \\
Saturated Fat & $270 \mathrm{mg}$ & 0.30 \\
Trans Fat & $60 \mathrm{mg}$ & 0.0 \\
Monounsaturated Fat & $0 \mathrm{mg}$ & $* *$ \\
Polyunsaturated Fat & $30 \mathrm{mg}$ & $* *$ \\
Cholesterol & $170 \mathrm{mg}$ & 0.0 \\
Total Carbohydrates & $0 \mathrm{mg}$ & $<2.0$ \\
Sugars & $60 \mathrm{mg}$ & $<2.0$ \\
Sugar Alcohols & $20 \mathrm{mg}$ & 0.0 \\
Other Carbohydrates & $0 \mathrm{mg}$ & $<2.0$ \\
Dietary Fiber & $40 \mathrm{mg}$ & 0.1 \\
Sodium (as sodium chloride) & $60 \mathrm{mg}$ & $<2.0$ \\
Protein & $160 \mathrm{mg}$ & 0.0 \\
& $0 \mathrm{mg}$ & \\
\hline
\end{tabular}

*Daily values are based on a 2,000 calories per day diet 
**Daily values not established

${ }^{\#}$ NT Factor Lipids ${ }^{\circledR}$ is a registered trademark of Nutritional Therapeutics Inc., Commack, NY.

\section{Materials and Methods:}

The liquid supplement test product, NT Factor Lipids ${ }^{\circledR}$ (Nutritional Therapeutics, Inc., Commack, NY), is a patent-pending proprietary nutrient drink containing an exogenous source of polyunsaturated phosphatidyl lipids and other membrane phospholipids (Table 1). The supplement drink was made up and distributed into $2 \mathrm{oz}(60 \mathrm{ml})$ bottles, sealed, and the containers were used within a few days. Thus antioxidants were not added to the preparation. The participants took the suggested dose $(60 \mathrm{ml}$ liquid drink) all at once after completion of the PFS evaluation.

The PFS form is composed of 22 numerically scaled questions rated from 0 (no fatigue) to 10 (severe) fatigue [18]. These items measure four dimensions of fatigue: behavioral/severity (6 items); affective/meaning (5 items); sensory (5 items); and cognitive/mood (6 items). These are used to calculate four sub-scale/dimensional scores and the overall fatigue levels of subjects. The standardized alpha (Cronbach's alpha) did not drop below 0.90 for any of the subscales, and the standard alpha for the entire scale of 22 questions was 0.96 , indicating excellent reliability for an established, validated instrument [20, 21].

The Supplemental Questionnaire Form was composed of 14 yes/no questions and 4 multiple-choice questions (Table 2). This form was administered along with the second PFS form at the end of the session to determine the time required for any improvements and to confirm any perceived improvements in cognitive function, mental clarity or focus.

Table 2. Supplementary Questionnaire Form

Based on your perception on the way you feel now, after you have taken the supplement drink, in comparison to before you took the supplement drink, please answer the following questions.

Percent Positive

$\begin{array}{lll}\text { Number } & \text { Question } & \text { Response }\end{array}$

1 Have you experienced a surge or feeling of energy and more vitality after taking the supplement drink?

2 How much more energy do you feel after taking the supplement drink?

$\begin{array}{lr}0 \% \text { increase } & 29 \\ 25 \% \text { increase } & 29 \\ 50 \% \text { increase } & 22 \\ 75 \% \text { increase } & 11 \\ 100 \% \text { increase } & 2\end{array}$

3 Do you think you are better able to face your necessary activities for the rest of the day?

4 Do you feel that you can participate in a better capacity in physical activities? 
5 Do you feel you have the energy and desire to participate in your favorite sport?

6 Do you feel you may have more stamina for activities or sports after taking this supplement drink?

7 Do you think you have more of a desire to increase your exercise level?

8 Do you think you are able to engage in conversation more effectively?

9 Do you feel that you are better able to plan tomorrow's activities?

10 Do you feel that you are better able to do your work at home or at the office?

11 Do you feel you have improved mental clarity?

12 Do you feel you have improved mental focus?

13 Do you think you are able to concentrate better?

14 If you feel an improvement in your mental focus, do you think your competitive edge in sports would be increased after taking this supplement drink?

15 Would you use this supplement increase your energy for participation in a particular activity?

16 Would you recommend this supplement to your friends and family? Not at all 26 Very likely 41

Most definitely 33

17 Please rate the taste of the supplement.

$\begin{array}{lc}\text { Poor } & 0 \\ \text { Fair } & 19 \\ \text { Good } & 70 \\ \text { Excellent } & 11\end{array}$

18 Approximately, how long did it take for you to feel increased energy? 15 min $\quad 25$

$30 \mathrm{~min} \quad 7$

$45 \min \quad 21$

$1 \mathrm{hr} \quad 18$

$2 \mathrm{hr}$ or more $\quad 0$

I did not feel a surge of energy 29

\section{Results:}

The glycophospholipid liquid supplement used in this study (Table 1) improved the overall fatigue scores of participants as measured by the PFS form by the end of the study (Table 3). 
The initial PFS group average (mean \pm standard error mean) total fatigue score was $3.35 \pm 0.27$, and after the study period this improved to $2.03 \pm 0.28$ or a $39.6 \%$ reduction in fatigue by the end of the session. The mean decrease in fatigue value was significant by t-test $(\mathrm{p}<0.0001)$ and Wilcoxon signed-rank $(p<0.0001)$ analyses. There were no adverse events during the course of the study. Although there were slight differences between the response in males and females, since the number of males was not similar to the number of females, we did not calculate these data separately.

\section{Table 3. Results From Overall Fatigue and Subcategories of the Piper Fatigue Scale Survey}

\begin{tabular}{|c|c|c|c|c|}
\hline \multirow[b]{2}{*}{ Category } & \multicolumn{2}{|c|}{ Mean Fatigue Level \pm S.E.M. } & \multirow{2}{*}{$\begin{array}{r}\text { Percent } \\
\text { Reduction }\end{array}$} & \multirow{2}{*}{$\begin{array}{c}\mathrm{t} \text {-test } \\
\mathrm{p}\end{array}$} \\
\hline & Hour 0 & Hour 3 & & \\
\hline Overall Fatigue & $3.35 \pm 0.27$ & $2.03 \pm 0.28$ & 39.6 & $<0.0001$ \\
\hline Behavior/Severity & $2.67 \pm 0.10$ & $1.29 \pm 0.10$ & 40.5 & $<0.0001$ \\
\hline Affective/Meaning & $3.18 \pm 0.14$ & $1.84 \pm 0.06$ & 42.1 & $<0.0003$ \\
\hline Sensory & $3.94 \pm 0.30$ & $2.84 \pm 0.67$ & 27.9 & $<0.0744$ \\
\hline Cognitive/Mood & $3.62 \pm 0.11$ & $1.83 \pm 0.11$ & 49.4 & $<0.0001$ \\
\hline
\end{tabular}

Since fatigue is a multidimensional phenomenon, the PFS evaluation can be further dissected into subcategories that include: overall fatigue, behavior/severity, affective meaning, sensory and cognitive/mood (Table 3) [18, 19]. All of these subcategories showed significant mean reductions at the end of the trial: $40.5 \%$ reduction $(\mathrm{p}<0.0001)$ in the Behavior/Severity category, $42.1 \%$ reduction $(\mathrm{p}<0.0003)$ in the Affective/Meaning category, $27.9 \%$ reduction $(p<0.0744)$ in the Sensory category and $49.4 \%$ reduction $(p<0.0001)$ in the Cognitive/Mood category. This indicated that there were improvements in all subcategories of fatigue. The cognitive/mood improvements indicated that there were perceived improvements in cognition and ability to concentrate, remember and think clearly after taking the NT Factor Lipids ${ }^{\circledR}$ energy drink (Table 3).

A supplemental survey form was also used to analyze subjects' perceptions of the taste, energy and mood effects of the NT Factor Lipids ${ }^{\circledR}$ energy drink (Table 2). A vast majority of the participants indicated that they perceived improvements in energy, vitality and stamina as well as improvements in mental clarity, focus and concentration, correlating well with the PFS data (Table 3). There was also general acceptance of the NT Factor Lipids® energy drink, and most would recommend the drink to friends and family members. Most participants felt these 
improvements within one hour after taking the liquid food supplement, and some subjects reported improvements within 15 min (Table 2).

\section{Discussion:}

The polyunsaturated glycophospholipid supplement in NT Factor ${ }^{\circledR}$ has been used successfully in animal and clinical lipid replacement studies [9, 10, 16, 20, 22]. In this formulation encapsulated lipids are protected from oxidation in the gut and can be absorbed and transported into tissues without oxidative damage $[9,10]$. The NT Factor ${ }^{\circledR}$ lipids are primarily membrane phospholipids and phosphoglycolipids that are normally found in vertebrate cellular membranes.

NT Factor ${ }^{\circledR}$ lipids have been used to prevent hearing loss associated with aging in rodents. In these studies NT Factor ${ }^{\circledR}$ lipids prevented hearing loss associated with aging and shifted the threshold hearing from 35-40 dB in control aged animals to $13-17 \mathrm{~dB}$ in the treatment group $(p<0.005)$ [23]. These authors also found that NT Factor ${ }^{\circledR}$ lipids preserved cochlear mitochondrial function, increasing mitochondrial function by 34\%. NT Factor ${ }^{\circledR}$ lipids also prevented aging-related mitochondrial DNA deletions found in the cochlear during the study [23].

In addition to aging, mitochondrial dysfunction and the accumulation of damaged mitochondrial components have been linked to a wide variety of chronic, metabolic and degenerative diseases and cancer [24, 25]. Lipid Replacement has been successfully used in clinical studies to reduce fatigue, increase mitochondrial function and protect cellular and mitochondrial membranes from oxidative damage [reviewed in 2, 24]. In multiple clinical studies fatigue was reduced $35-43 \%$ by oral administration of NT Factor ${ }^{\circledR}$ [10, 16, 20, 22]. Even in severely fatigued patients with chronic fatigue syndrome or fibromyalgia syndrome, NT Factor ${ }^{\circledR}$ reduced fatigue by $43.1 \%$ [10]. In the study by Agadjadyan et al. [22] NT Factor ${ }^{\circledR}$ reduced fatigue $35.5 \%$ in aging adults and significantly improved mitochondrial function to a level that was similar to that found in young, healthy adults. Thus NT Factor ${ }^{\circledR}$ has the potential to decrease the effects of aging on mitochondrial function and improve mitochondrial function in chronic diseases.

In different NT Factor ${ }^{\circledR}$ combinations (such as $\operatorname{Propax}^{\mathrm{TM}}$ with NT Factor ${ }^{\circledR}$ plus antioxidants, vitamins and minerals) fatigue was significantly reduced in cancer patients. In addition, Propax ${ }^{\mathrm{TM}}$ with NT Factor ${ }^{\circledR}$ reduced the adverse effects of cancer therapy, such as chemotherapy-induced fatigue, nausea, vomiting and other side effects associated with chemotherapy [26, 27]. This double-blinded, cross-over, placebo-controlled, randomized trial on advanced cancer patients receiving chemotherapy showed that $\operatorname{Propax}^{\mathrm{TM}}$ with NT Factor ${ }^{\circledR}$ improved fatigue, nausea, diarrhea, impaired taste, constipation, insomnia and other quality of life indicators [27].

In the present study we used a new LRT preparation of NT Factor Lipids ${ }^{\circledR}$ (without probiotics, minerals or vitamins) in a liquid drink to improve fatigue and mental function. In this formulation fatigue as measured by the validated PFS instrument was reduced by a mean of $39.6 \%$ within three hours. These subjects also experienced perceived improvements in energy, vitality and stamina as well as perceived improvements in mental clarity, focus and concentration. Although this study was not placebo-controlled, it is unlikely that placebo effects 
could account for the reductions in fatigue and perceived improvements in mental function. Moreover, the results were comparable to previous studies using NT Factor ${ }^{\circledR}$ where fatigue was reduced by $35-43 \%$ as assessed using a validated instrument for monitoring fatigue [10, 16, 20, 22].

Finally, the possible role of probiotics that are usually present in NT Factor® formulations could not be assessed here. Previously we speculated that probiotics might improve the digestion and intestinal transport of NT Factor ${ }^{\circledR}$ lipids; however, probiotics were not in the formulation used here, and the improvements in fatigue and mental function occurred rather quickly. This may be attributed to the fact that NT Factor Lipids® were administered in a $60 \mathrm{ml}$ liquid drink, and the lipid formulation used in NT Factor Lipids® has been improved over the years since NT Factor® was first introduced. These preliminary results indicate that NT Factor Lipids $\circledast$ alone can reduce fatigue and improve perceived mental function.

Most liquid energy drinks contain sugar, caffeine and/or herbs or other stimulants [7, 8]. Stimulants, such as caffeine, can cause hypertension, heart palpitations, headaches and insomnia [28]. NT Factor Lipids ${ }^{\circledR}$ does not contain these components. Thus functional food "energy" drinks can be developed that do not contain sugar, stimulants or herbs. This study indicates that such liquid products are effective in reducing fatigue and improving the perception of increased energy and ability to concentrate, remember and think clearly.

\section{Competing Interests:}

The authors have no financial interests or conflicts of interest.

\section{Authors' Contributions:}

All authors contributed to this study.

\section{Abbreviations:}

LRT, Lipid Replacement Therapy; PFS, Piper Fatigue Scale; ROS/RNS, reactive oxygen and nitrogen species

\section{Acknowledgements and Funding:}

The authors would like to thank Nutritional Therapeutics, Inc. and the Institute for Molecular Medicine for clinical trial financial support. We also thank Mr. Talon Ellithorpe and Mr. Rob Fielding for expert assistance.

\section{References:}

1. Kroenke K, Wood DR, Mangelsdorff AD, Meier NJ, Powell JB. Chronic fatigue in primary care. Prevalence, patient characteristics, and outcome. JAMA 1988; 260: 929-934.

2. Nicolson GL, Settineri R. Lipid Replacement Therapy: a functional food approach with new formulations for reducing cellular oxidative damage, cancer-associated fatigue and the adverse effects of cancer therapy. Funct Foods Health Dis 2011; 4: 135-160.

3. Morrison JD. Fatigue as a presenting complaint in family practice. J Family Pract 1980; 10: 795-801. 
4. Knicker AJ, Renshaw I, Oldham AR, Cairns SP. Interactive processes link the multiple symptoms of fatigue in sports competition. Sports Med 2011; 41: 307-328.

5. Purvis D, Gonsalves S, Deuster PA. Physiological and psychological fatigue in extreme conditions: overtraining and elite athletes. Phys Med Rehab 2010; 2: 442-450.

6. Sheaff AK, Bennett A, Hanson ED, Kim YS, Hsu J, Shim JK, Edwards ST, Hurley BF. Physiological determinants of the candidate physical ability test in firefighters. J Strength Cond Res 2010; 24: 3112-3122.

7. Heckman MA, Weil J, Gonzalez de Mejia E. Caffeine (1,3,7-trimethulxanthine) in foods: a comprehensive review on consumption, functionality, safety and regulatory matters. J Food Sci 2010; 75: R77-R87.

8. Wang J, Li S, Fan Y, Chen Y, Liu D, Cheng H, Zhou Y. Anti-fatigue activity of the water soluble polyscaccharides isolated from Panax ginseng C. A. Meyer. J Ethnopharmacol 2010; 30: 421-423.

9. Nicolson GL. Lipid replacement as an adjunct to therapy for chronic fatigue, anti-aging and restoration of mitochondrial function. J Am Nutraceut Assoc 2003; 6(3): 22-28.

10. Nicolson, G.L. and Ellithrope, R. Lipid replacement and antioxidant nutritional therapy for restoring mitochondrial function and reducing fatigue in chronic fatigue syndrome and other fatiguing illnesses. J Chronic Fatigue Syndr. 2006; 13(1): 57-68.

11. Kanno T, Sato EE, Muranaka S, Fujita H, Fujiwara T, Utsumi T, Inoue M, Utsumi K. Oxidative stress underlies the mechanism for $\mathrm{Ca}(2+)$-induced permeability transition of mitochondria. Free Radical Res. 2004; 38(1): 27-35.

12. Huang $\mathrm{H}$, Manton KG. The role of oxidative damage in mitochondria during aging: a review. Front Biosci. 2004; 9: 1100-1117.

13. Halliwell B. Oxidative stress, nutrition and health. Free Radical Res 1996; 25: 57-74.

14. Stadtman E. Introduction to serial reviews on oxidatively modified proteins in aging and disease. Free Radical Biol Med 2002; 32: 789.

15. Castro L, Freeman BA. Reactive oxygen species in human health and disease. Nutr 2001; 17: 295-307.

16. Huang JH, Hood DA. Age-associated mitochondrial dysfunction in skeletal muscle: contributing factors and suggestions for long-term interventions. IUBMB Life 2009; 61: 201-214.

17. Ellithorpe RR, Settineri R, Nicolson GL. Pilot study: reduction of fatigue by use of a dietary supplement containing glycophospholipids. J Am Nutraceut Assoc. 2003; 6(1): 2328.

18. Piper BF, Dribble SL, Dodd MJ. The revised Piper Fatigue Scale: psychometric evaluation in women with breast cancer. Oncol Nursing Forum 1998; 25: 667-684.

19. Piper BF, Linsey AM, Dodd MJ. Fatigue mechanism in cancer. Oncol Nursing Forum 1987; 14: 17-23.

20. Nicoson GL, Ellithorpe RR, Ayson-Mitchell C, Jacques B, Settineri R. Lipid Replacement Therapy with a glycophospholipid-antioxidant-vitamin formulation significantly reduces fatigue within one week. J Am Nutraceutical Assoc 2010; 13(1): 11-15.

21. Nunnally JC. Psychometric Theory, $2^{\text {nd }}$ ed., New York: McGraw-Hill. 1978, pp. 117-123. 
22. Agadjanyan M, Vasilevko V, Ghochikyan A, Berns P, Kesslak P, Settineri R, Nicolson GL. Nutritional supplement (NTFactor) restores mitochondrial function and reduces moderately severe fatigue in aged subjects. J Chronic Fatigue Syndr 2003; 11(3): 23-26.

23. Seidman M, Khan MJ, Tang WX, Quirk WS. Influence of lecithin on mitochondrial DNA and age-related hearing loss. Otolaryngol Head Neck Surg 2002; 127: 138-144.

24. Wallace DC. A mitochondrial paradigm of metabolic and degenerative diseases, aging and cancer: a dawn for evolutionary medicine. Annu Rev Genet 2005; 39: 359-407.

25. Wallace DC, Fan W, Procaccio V. Mitochondrial energetics and therapeutics. Annu Rev Pathol 2010; 5: 297-348.

26. Nicolson GL. Lipid Replacement Therapy: a nutraceutical approach for reducing cancerassociated fatigue and the adverse effects of cancer therapy while restoring mitochondrial function. Cancer Metastasis Rev 2010; 29: 543-552.

27. Colodny L, Lynch K, Farber C, Papish S, Phillips K, Sanchez M, Cooper K, Pickus O, Palmer D, Percy TB, Faroqui M, Block JB. Results of a study to evaluate the use of Propax to reduce adverse effects of chemotherapy. J Am Nutraceut Assoc 2000; 3(1): 17-25.

28. Steinke L, Lafear DE, Dhanapal V, Kalus JS. Effect of "energy drink" consumption on hemodynamic and electrocrdiographic parameters in healthy young adults. Ann Pharmacother 2009; 43: 596-602. 